\title{
ArcheoSciences
}

Revue d'archéométrie

\section{Le travail du fer dans l'établissement perché tardo- antique du Roc de Pampelune (Argelliers, Hérault) : l'apport des analyses métallographiques}

\section{Gaspard Pagès, Laurent Schneider et Philippe Fluzin}

\section{(2) OpenEdition}

Journals

Édition électronique

URL : https://journals.openedition.org/archeosciences/569

DOI : 10.4000/archeosciences.569

ISBN : 978-2-7535-1594-9

ISSN : 2104-3728

\section{Éditeur}

Presses universitaires de Rennes

\section{Édition imprimée}

Date de publication : 31 décembre 2005

Pagination : 107-116

ISSN : 1960-1360

Référence électronique

Gaspard Pagès, Laurent Schneider et Philippe Fluzin, « Le travail du fer dans l'établissement perché tardo-antique du Roc de Pampelune (Argelliers, Hérault) : l'apport des analyses métallographiques », ArcheoSciences [En ligne], 29 | 2005, mis en ligne le 31 décembre 2007, consulté le 28 janvier 2022. URL : http://journals.openedition.org/archeosciences/569; DOI : https://doi.org/10.4000/ archeosciences.569 


\title{
Le travail du fer dans l'établissement perché tardo-antique du Roc de Pampelune (Argelliers, Hérault) : l'apport des analyses métallo- graphiques
}

\author{
Gaspard PAGĖS*, Laurent SCHNEIDER** et Philippe FLUZIN***
}

\begin{abstract}
Résumé : Le site perché du Roc de Pampelune occupe, de la fin du V' siècle au milicu du VI siècle, un plateau calcaire dans la garrigue nord montpelliéraine (Hérault, France) sur un peu plus de deux hectares. Depuis 1999, sa fouille a livré plus de 5400 scories (soit environ $109 \mathrm{~kg}$ ) et de nombreuses structures métallurgiques qui ont motivé un examen approfondi pour localiser les espaces de travail et préciser le type d'activité. Une lecture croiséc liant analyses archéologiques et métallographiques a donc été réaliséc afin de déterminer le rôle ct la place de la métallurgic au scin de cet ćtablissement. Ainsi, est mise en évidence, à côté du rôle administratif, l'importance économique de ces habitats perchés dans la réorganisation du réseau de peuplement des campagnes gauloises au cours du premier Moyen Age.

Abstract: The hill-top site of the "Roc de Pampclune" occupies, from the end of the Vth century A.D. to the middle of the VIth century, a limestonc plateau in the ilex oak forests north of Montpellier (Hérault, France) covering an area over two hectares. Since 1999, the excavation of this site has produced more than 5400 fragments of slag (amounting to $109 \mathrm{~kg}$ ) and many metallurgical structures which have led to extensive research in order to pinpoint work areas and to establish the kind of production. Conjoint archaeological and metallographic analyses have been conducted in order to determine the part and place of metallurgy in this settlement. This way, we have underlined the economic importance as well as the administrative part of these hill-top sites in the reorganisation of the setticment pattern of the gaulish countryside during the first phase of the Middle-Ages.
\end{abstract}

Mots-clefs : Métallurgie du fer, forgeage, demi-produit, habitat perché, Antiquité tardive.

Key-words: Iron mctallurgy, forging, half-product, hill-top site, Late Antiquity.

Le Roc de Pampelune (Argelliers, l'Hérault) fait l'objet depuis 1999 d'une exploration archéologique extensive dans le cadre d'une Action Thématique Incitative sur Programme du CNRS soutenue par le Ministère de la Culture (Schneider, 2003). Il appartient à une nouvelle génération de sites perchés et fortifiés qui émergent un peu partout dans le sud de la Gaule au cours des V-VII ${ }^{\circ}$ siècle. Longtemps considéré comme un type d'habitat refuge connoté d'un certain misérabilisme et associé au contexte des migrations des peuples européens à la fin de l'Antiquité, sept ans de fouilles permettent aujourd'hui de dénoncer une posture historiographique fondée sur une lecture sans doute trop hâtive des textes (Schneider, 2003). Les données accumulées au Roc de Pampelune livre désormais une image beaucoup plus complexe, celle d'une communauté d'une centaine d'habitants ras- semblée dans le dernier tiers du V siècle dans un espace forestier derrière une enceinte enveloppant 2,25 ha densément occupés (fig. 1 et 2). Encore ouverte sur le commerce méditerranéen (Orient et Afrique du nord), cette communauté dispose d'une église baptismale, réside et travaille dans des bâtiments maçonnés couverts de tuiles et doit être associée à une époque de forte mobilité rurale et de réorganisation des réseaux locaux du peuplement.

Dans ce contexte, l'importante proportion de déchets métallurgiques découverts (5476 scories pour $109 \mathrm{~kg}$ ) a motivé l'élaboration d'une étude distincte pour localiser les espaces de travail et définir la place de l'activité métallurgique au sein d'un artisanat polyvalent et de pratiques agro-pastorales plus traditionnelles. Des moyens spécifiques sont alors mis en cuvre en appliquant simultanément une démarche archéologique, répartition spatiale

\footnotetext{
* UMR 5140 - Archéologie des sociétés méditerranéennes : Milieux, territoires et civilisations - 390 av. de Pérols, 34970 LATTES; UMR 5060 Laboratoire Métallurgies et Cultures - UTBM. Site de Sévenans, 90010 BELFORT cedex. Email : gaspard.pages@)free.fr

** UMR 6572, Laboratoire d'Archéologie Médiévale Méditerranéenne - MMSH, 5 rue du Château de l'Horloge, 13094 ALX-EN-PROVENCE. Email : lauschne@club-internet.fr

*** UMR 5060 - Laboratoire Métallurgies et Cultures - UTBM Site de Sévenans, 90010 BELFORT cedex. Email : lmc(@utbm.fr
} 


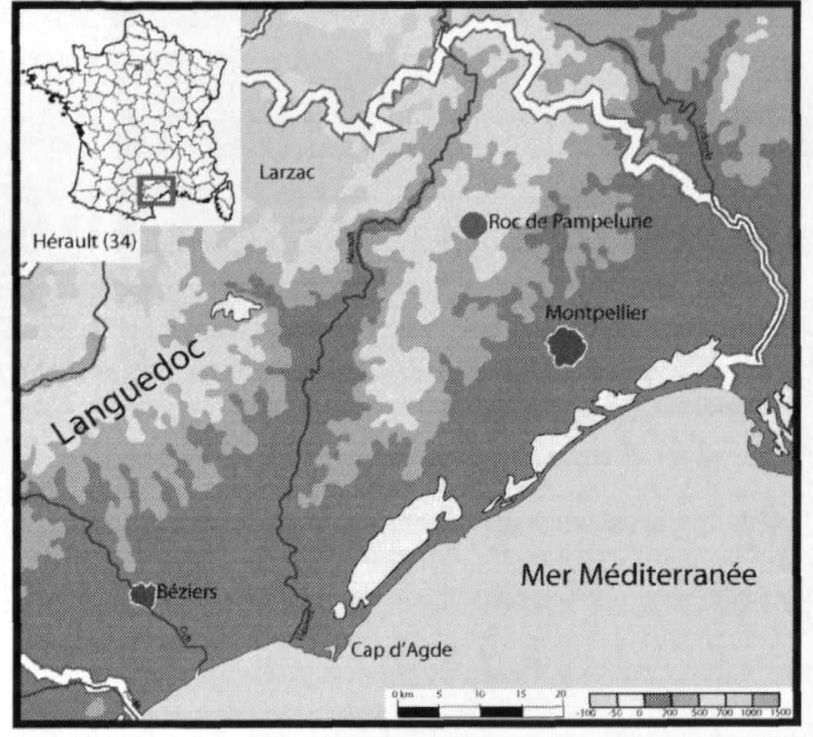

Fig. 1 : Localisation du site du Roc de Pampelune (Argelliers, Hérault) (réalisation UMR 5140, complété par Pagès).

Fig. 1: Map showing the location of the site of the Roc de Pampelune (Argelliers, Hérault) (drawn by UMR 5140, completed by Pagès).

et classement typologique ..., et des analyses métallographiques ${ }^{1}$, étude des structures et des propriétés des métaux. Le rôle et la place de la métallurgie au sein de cet établissement sont alors précisés et permettent d'aborder la question des changements techniques et économiques à la fin de l'Antiquité et plus particulièrement entre les années 475 et 550 ap. J.-C.

\section{Les structures et les déchets métallurgiques}

\subsection{Les foyers de forge}

La chênaie et le terrain très rocailleux ne favorisent pas la conservation des vestiges archéologiques. Ils sont généralement très perturbés, à l'image d'un foyer de forge surélevé établi sur une base en calcaire où sont disposés des blocs de grès rubéfiés. Identifié également grâce à la présence de scories de forge, cet atelier est localisé dans le quartier sommital de l'agglomération, dans la petite pièce (3D1) d'un bâtiment implanté à proximité de l'église baptismale qui clôture la cour (fig. 2).

Un second foyer de forge bouleversé est installé à l'autre extrémité de la bourgade, dans un espace ouvert (2A3) délimité par le rempart septentrional et un bâtiment (fig. 2). Il contient le plus important lot de scories et de nombreuses battitures uniformément réparties sans qu'il soit possible de déceler par empreinte négative la position d'une enclume ou des espaces de travail. Le foyer a été totalement détruit, il ne subsiste sur le substrat qu'une zone éclatée par la chaleur où de la scorie est incrustée.

Entre ces deux secteurs, dans la petite pièce (1L1) d'un bâtiment construit un peu en retrait du rempart septentrional, il existe de nombreuses scories disséminées dans tout l'espace, avec une concentration particulière au milieu de fragments de paroi de forge qui signalent l'existence d'un foyer également très endommagé (fig. 2).

Le seul foyer de forge $(38 \times 20 \times 12 \mathrm{~cm})$ correctement conservé est implanté dans des couches de construction d'un bâtiment (1S1) localisé au centre de la bourgade (fig. 2). La rubéfaction peu importante des parois et la faiblesse du nombre de scories associées confirment son utilisation temporaire, certainement liée à la construction des bâtiments environnants. Son creusement très charbonneux conserve en place une scorie en forme de culot recouverte par des fragments d'argile rubéfiée et scorifiée percée d'un trou de 3 centimètres de diamètre. Ils signalent l'existence d'un muret construit pour protéger le soufflet des rayonnements thermiques du foyer et pour aménager une tuyère destinée à la ventilation (fig. 3).

\subsection{Les outils du forgeron}

Malgré l'observation attentive des blocs de pierre avoisinants les structures métallurgiques, aucune enclume n'a pu être découverte. Un seul outil de forgeron a été mis au jour dans un épandage domestique constitué à l'arrière du rempart septentrional $(2 \mathrm{~L})$, en contrebas de la forge du bâtiment $1 \mathrm{~L}$ (fig. 2). Il s'agit d'un poinçon en fer (fig. 4) dont au moins quatre exemplaires similaires datés du $\mathrm{I}^{\mathrm{er}}$ au $\mathrm{III}^{\mathrm{e}}$ siècle après J.-C. sont conservés au Musée d'Avanches en Suisse (Duvauchelle, 2005). Cet outil percutant, servant à faire glisser la matière, est constitué d'un manche biconique de section carrée surmonté d'une tête en forme de champignon ( $90 \times 15 \times 15 \mathrm{~mm}$ pour environ $200 \mathrm{~g}$ ) (Fluzin, 1983). Occasionnellement, cette dernière pouvait probablement servir d'enclumette en plantant l'extrémité opposée dans un billot de bois. Cet outil est utilisé aussi bien dans le travail des métaux ferreux que dans celui des non ferreux.

\subsection{Les concentrations de scories}

Les scories sont principalement concentrées dans l'espace ouvert de l'angle nord oriental de l'agglomération, où des reliquats d'un foyer de forge sont incrustés sur le substrat ( $2 \mathrm{~A} 3: 2308$ scories pour $34 \mathrm{~kg}$ soit $42 \%$ et $31 \%$ de la totalité). Elles sont également massivement présentes dans les deux sondages de l'épandage sédimentaire constitué contre une enceinte primitive du quartier sommital qui sert alors de terrasse $(1 \mathrm{H}: 1309$ scories pour $21 \mathrm{~kg}$ soit $24 \%$ et $19 \%$ de la totalité). L'arasement des niveaux archéologiques de cette zone est perceptible dans le ravinement des déchets métallurgiques qui se retrouvent en contrebas dans les espaces ouverts $1 \mathrm{G}$ et $10: 248$ scories pour $14 \mathrm{~kg}$ soit $4 \%$ et $14 \%$ de la totalité). Un dernier lot important de scories est concentré autour des vestiges du foyer de forge mis au jour dans la petite pièce du bâtiment $1 \mathrm{~L}$ construit au centre de l'établissement $\left(1 \mathrm{~L}, 1 \mathrm{Q}^{2}, 1 \mathrm{~L}\right.$, et $1 \mathrm{~T}$ : 761 scories pour $11 \mathrm{~kg}$ soit $14 \%$ et $10 \%$ de la totalité) (fig. 2).

Les grands ensembles de scories ont été découverts dans des formations sédimentaires de teinte sombre,

\footnotetext{
${ }^{1}$ Les analyses métallographiques ont été réalisées en 2002, 2003 et 2004 au sein de l’UMR 5060 “ Laboratoire Métallurgies et Cultures ” UTBM Site de Sévenans 90010 Belfort.

${ }^{2}$ Le bâtiment $1 Q$ est une construction légèrement postérieure qui s'appuie sur les sols de circulation des extérieurs du bâtiment $1 \mathrm{~L}$.
} 


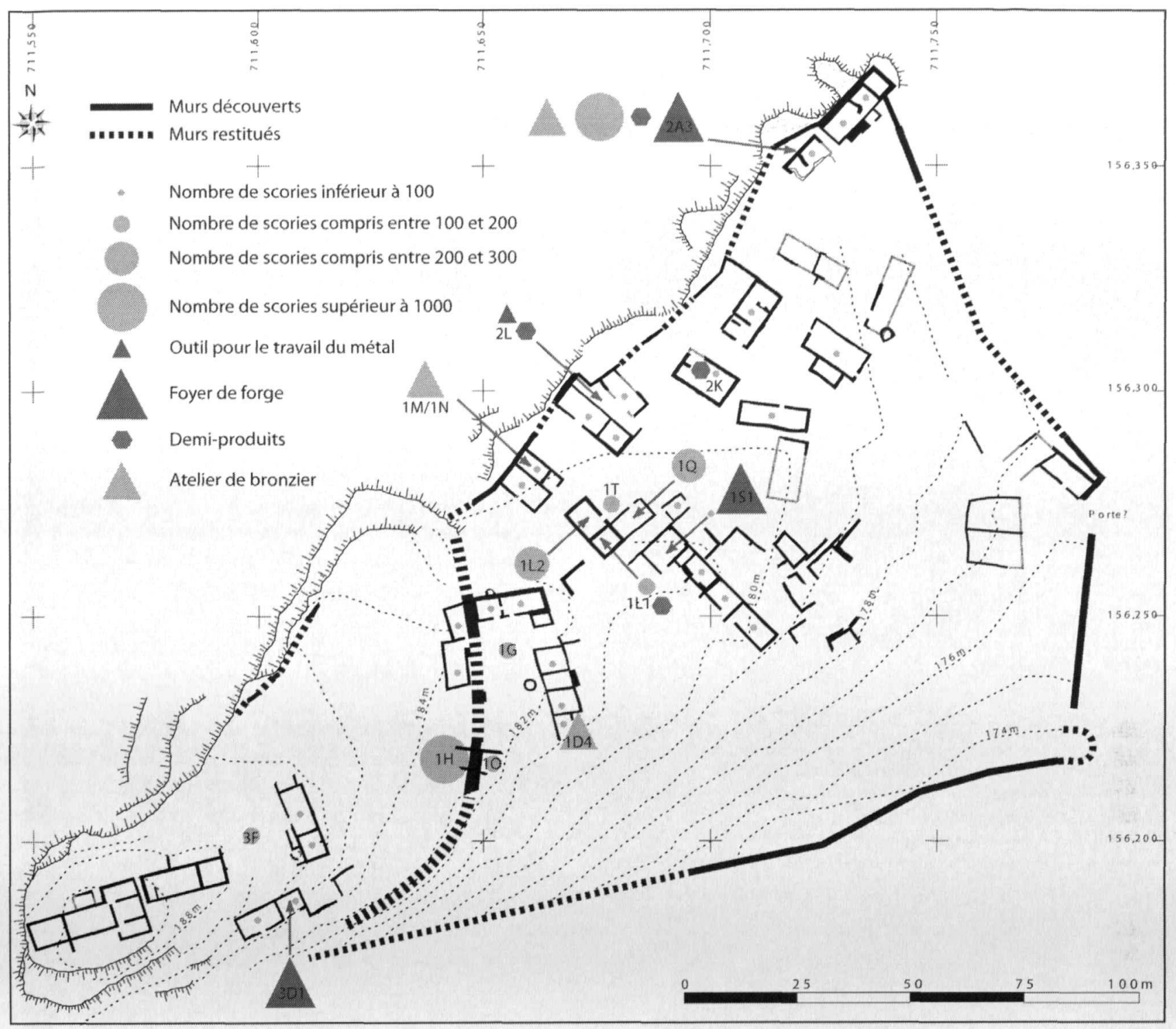

Fig. 2 : Plan général du site du Roc de Pampelune (Argelliers, Hérault) et répartition des vestiges métallurgiques (Réalisation Schneider et Marchand, 2004, complété par Pagès).

Fig. 2: Global map of the Roc de Pampelune site (Argelliers, Hérault) and of metallurgical artefact distribution (drawn by Schneider and Marchand, 2004, completed by Pagès).

souvent pulvérulentes, riches également en fragments de céramiques. La formation de ces nombreux épandages, typiques des espaces ouverts, est certainement à mettre en relation avec l'activité métallurgique et plus généralement avec l'ensemble des arts du feu mis en évidence sur le site comme cela a été démontré par exemple sur la colline voisine de Mas-Viel ou s'installe une industrie potière entre la fin du $\mathrm{XII}^{\mathrm{c}}$ et la première moitié du XIIII ${ }^{\mathrm{e}}$ siècle (Breichner et al., 2002). On ne peut manquer cependant de les associer plus largement à la problématique des «terres noires» que l'on observe dans les villes du nord de la Gaule et qui témoignent de nouvelles conditions de vie dans les anciens centres urbains (Cammas et al., 1995 ; Guyard, 2003). L'apparition de ces sédiments spécifiques de la fin de l'Antiquité commence également a être notée dans des villae et de petites agglomérations du Languedoc (Pellecuer, 2000).

\section{Les déchets métallurgiques et leur analyse métallographique}

\subsection{Les déchets métallurgiques}

Le lot de scories issu des trois zones ressort de types différenciés définis selon une terminologie commune (Mangin, 2004; Fluzin et al., 2000; Serneels, 1998) (tab. 1). Les déchets provenant des épandages de la terrasse $1 \mathrm{H}$ sont essentiellement constitués de culots de forge (42 individus pour $7200 \mathrm{~g}$ ) et de scories informes denses (1058 individus pour $12300 \mathrm{~g}$ ), tandis qu'à l'opposé ceux de la cour $2 \mathrm{~A} 3$ se distinguent par leur forte proportion en scories coulées peu denses (426 individus pour $3800 \mathrm{~g}$ ), en gromps ${ }^{3}$ (34 individus pour $1790 \mathrm{~g}$ ) et en parois scorifiées (145 individus pour $1440 \mathrm{~g}$ ). Ce type d'assemblage, comprenant également de nombreux

\footnotetext{
${ }^{3}$ Gromps : terme polonais désignant un déchet associé à la première épuration de la masse de fer brute (massiau ou loupe) et aux diverses phases de compactage postérieures. Ils sont principalement caractérisés par leur forte proportion de métal peu épuré enveloppé dans une gangue scorifiée informe.
} 


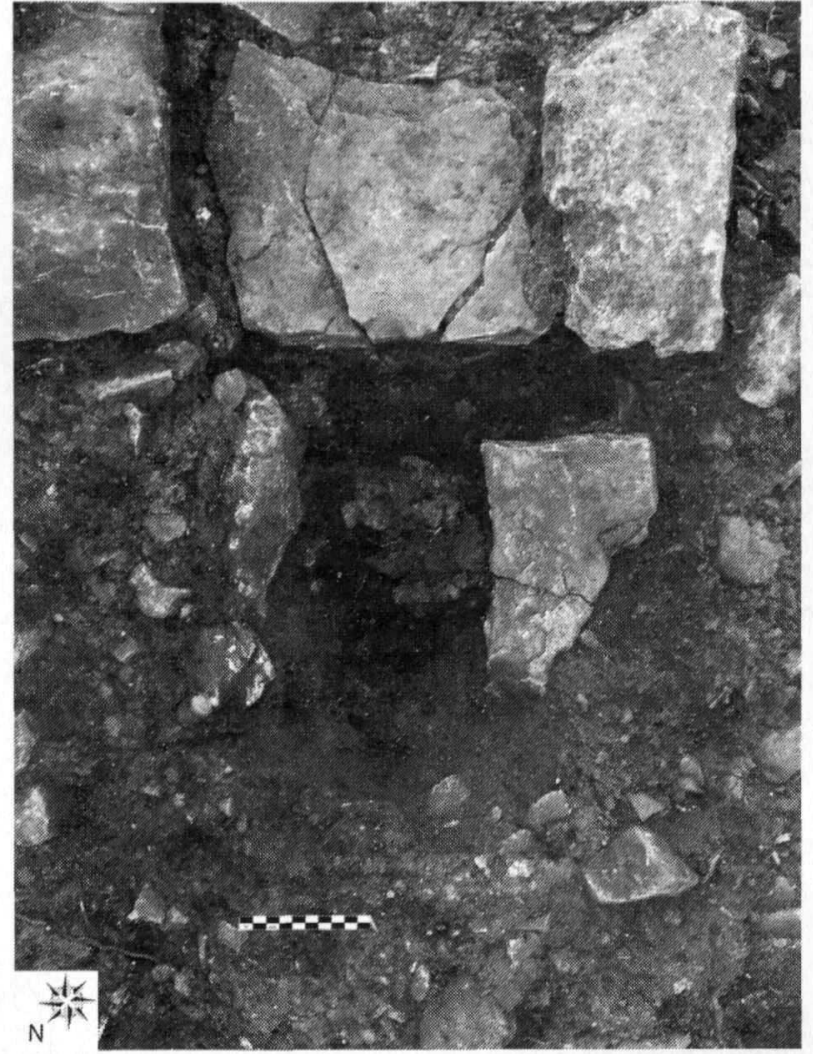

Fig. 3 : Foyer de forge temporaire creusé dans le bâtiment 1S1. Au sud, le mur de la construction recoupe la structure de combustion, au centre de laquelle se trouve le culot de forge.

Fig. 3: Temporary smelting furnace dug in the building ISI. In the south, the wall of construction recuts the structure of combustion where is the ingot solidified at the bottom of the smelting furnace.

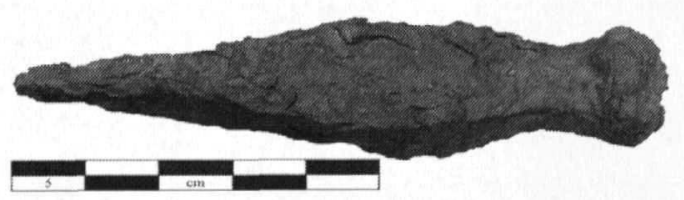

Fig. 4 : Poinçon en fer destiné au travail des métaux. Fig. 4: Style or hallmark intended for metal working. culots (24 individus pour $4950 \mathrm{~g}$ ), est sensiblement équivalent au lot de l'atelier implanté dans $1 \mathrm{~L} 1$.

L'hétérogénéité des lots de déchets invite à réaliser des analyses métallographiques distinctes sur chaque ensemble de manière à mieux connaître les activités métallurgiques exercées. La similitude des lots provenant de 1L1 et de $2 \mathrm{~A} 3$ et leur ressemblance morphologique incitent toutefois à une analyse commune corrigée par l'examen de certains éléments exceptionnels et caractéristiques.

Les analyses métallographiques sont fondées sur l'observation d'une surface polie au micron d'un échantillon de scorie ou d'objet en fer à l'aide d'un microscope optique et d'une lumière polarisée. La structure du matériau est ainsi examinée pour connaître l'aspect, la disposition et la forme des grains de métal et d'oxyde. En fonction des critères observés, les techniques de la chaîne opératoire - réduction, épuration, forgeage... - et de mise en œuvre peuvent être caractérisées. Dans ce programme, 19 échantillons ont été examinés. Ils sont répartis de la manière suivante : 11 culots de forge, 4 demi-produits, 4 objets en cours de confection (tab. 2).

\subsection{Analyse métallographique des scories de l'épan- dage $1 \mathrm{H}$}

L'épandage $1 \mathrm{H}$ correspond à une formation sédimentaire mêlant activités domestiques et métallurgiques, il est donc difficile de prélever des objets métalliques susceptibles de provenir de l'activité métallurgique génératrice des déchets $(1 \mathrm{H}: 43 \%$ de céramique, $33 \%$ d'os et coquillage, $19 \%$ de scorie, $3 \%$ de verre et $2 \%$ d'objet en fer). Pour cette raison, ce sont 7 scories en forme de culot, déchets caractéristiques des activités de forge, qui ont été analysées (échantillons 1389.1, 2, 3; 1393.2, 3 ; $1409.3,4)$. Elles dévoilent une matrice fayalitique ${ }^{4}$ équivalente mais très hétérogène avec de la wustite ${ }^{5}$ sous forme globulaire provenant essentiellement de battitures ${ }^{6}$ plates et globulaires (fig. 5). Elles se distinguent également par la présence importante de gros morceaux de métal ou de fragments déchiquetés déjà très bien épurés

\begin{tabular}{|c|c|c|c|c|c|c|c|c|c|}
\hline & $\begin{array}{c}\text { Ensembles } \\
\text { stratigraphiques }\end{array}$ & & $\mathbf{A} 3$ & & H & & $1 \mathrm{~L}$ & & D \\
\hline & Types & nbre & $\begin{array}{l}\text { poids } \\
(\mathrm{g})\end{array}$ & nbre & $\begin{array}{c}\text { poids } \\
(\mathrm{g})\end{array}$ & nbre & \begin{tabular}{|c|} 
poids \\
$(\mathrm{g})$
\end{tabular} & nbre & $\begin{array}{l}\text { poids } \\
(\mathrm{g})\end{array}$ \\
\hline & coulées peu denses & 426 & 3800 & 61 & 350 & 0 & 0 & 17 & 200 \\
\hline & en forme culot & 24 & 4950 & 42 & 7200 & 18 & 1750 & 7 & 1300 \\
\hline & denses informes & 1077 & 17700 & 1058 & 12300 & 233 & 2550 & 58 & 1400 \\
\hline 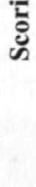 & $\begin{array}{l}\text { très peu denses, de } \\
\text { forme molle, non } \\
\text { magnétiques et } \\
\text { généralement } \\
\text { vitrifiées }\end{array}$ & 602 & 4300 & 93 & 510 & 38 & 200 & 0 & 0 \\
\hline & ragments de paroi & 145 & 1440 & 41 & 200 & 48 & 400 & 12 & 200 \\
\hline & Gromps & 34 & 1790 & 14 & 600 & 9 & 250 & 0 & 0 \\
\hline & Total & 2308 & 33980 & 1309 & 21160 & 346 & 5150 & 94 & 3100 \\
\hline
\end{tabular}

Tab. 1 : Répartition numéraire et pondérale des scories découvertes dans les principaux ensembles métallurgiques. Table 1: Numerical and weighted distribution of slag found in the main metallurgical sets. 


\begin{tabular}{|c|c|c|c|c|c|}
\hline$\stackrel{气}{\tilde{E}}$ & $\mathbf{N}^{\circ}$ & Types & $\begin{array}{l}\text { Dimensions: } \\
\text { Lxlxh (mm) }\end{array}$ & Poids (g) & Conclusions métallographiques \\
\hline $1 \mathrm{~L} 1$ & 1176.1 & Demi-produit & $75 \times 52 \times 27$ & 190 & $\begin{array}{l}\text { Loupe en cours d'épuration abandonnée dans le foyer de forge. } \\
\text { Constitution : aciérée ( } 100 \% \text { dont } 80 \% \text { eutectoöde). }\end{array}$ \\
\hline \multirow{7}{*}{$1 \mathrm{H}$} & 1389.1 & \multirow{7}{*}{ Culot de forge } & $97 \times 81 \times 42$ & 456 & $\begin{array}{l}\text { Cycles thermiques courts et hétérogènes réalisés à basses } \\
\text { températures. Peu de pertes de métal. }\end{array}$ \\
\hline & 1389.2 & & $97 \times 68 \times 42$ & 362 & $\begin{array}{l}\text { Cycles thermiques courts et hétérogènes réalisés à basses } \\
\text { températures. Peu de pertes de métal. }\end{array}$ \\
\hline & 1389.3 & & $79 \times 62 \times 31$ & 184 & $\begin{array}{l}\text { Cycles thermiques homogènes réalisés à haute température. } \\
\text { Beaucoup de pertes de métal sous forme de fragments et de filaments. }\end{array}$ \\
\hline & 1393.2 & & $81 \times 65 \times 38$ & 202 & $\begin{array}{l}\text { Cycles thermiques courts et hétérogènes réalisés à basses } \\
\text { températures. Beaucoup de pertes de métal sous forme de fragments } \\
\text { et de filaments. }\end{array}$ \\
\hline & 1393.3 & & $95 \times 74 \times 27$ & 254 & $\begin{array}{l}\text { Cycles thermiques courts et hétérogènes réalisés à basses } \\
\text { températures. Bcaucoup de pertes de métal sous forme de fragments, } \\
\text { de filaments et de gros morceaux ferritiques, épurés et écrouis. }\end{array}$ \\
\hline & 1409.2 & & $78 \times 68 \times 33$ & 196 & $\begin{array}{l}\text { Cycles thermiques homogènes réalisés à haute température. } \\
\text { Bcaucoup de pertes de métal sous forme de fragments et de filaments. }\end{array}$ \\
\hline & 1409.4 & & $81 \times 63 \times 30$ & 176 & $\begin{array}{l}\text { Cycles thermiques courts et hétérogènes réalisés à basses } \\
\text { températures. Beaucoup de pertes de métal sous forme de fragments } \\
\text { et de filaments. }\end{array}$ \\
\hline $\mathbf{2 H}$ & 2505.1 & Demi-produit & $43 \times 36 \times 11$ & 110 & $\begin{array}{l}\text { Issu d'une phase de martelage. Bonne propreté inclusionnaire ct taux } \\
\text { de compactage élevé. Constitution : aciérée ( } 70 \% \text { dont } 90 \% \\
\text { eutectoöde) et ferritique (30\%). }\end{array}$ \\
\hline $2 \mathrm{~K}$ & 2528.1 & Assemblage de tôle & $42 \times 37 \times 10$ & 40 & $\begin{array}{l}\text { Issu d'une phase de martelage. Bonne propreté inclusionnaire et taux } \\
\text { de compactage très élevé mais soudure mal réalisée. Constitution : } \\
100 \% \text { ferritique. }\end{array}$ \\
\hline $2 \mathrm{~L}$ & 2535.1 & Demi-produit & $157 \times 26 \times 10$ & 3.38 & $\begin{array}{l}\text { Très bonne propreté inclusionnaire et taux de compactage élevé à } \\
\text { l'exception d'une extrémité. Constitution : aciérée (90\% dont } 90 \% \\
\text { eutectoide ct trempé) et ferritique ( } 10 \%) \text {. }\end{array}$ \\
\hline \multirow{7}{*}{$2 \mathrm{A3}$} & 2180.1 & \multirow{3}{*}{ Culot de forge } & $143 \times 121 \times 95$ & 1340 & $\begin{array}{l}\text { Cycles thermiques homogènes réalisés à haute température. Peu de } \\
\text { pertes de métal. Double culot. }\end{array}$ \\
\hline & 2180.2 & & $120 \times 75 \times 40$ & 332 & $\begin{array}{l}\text { Cycles thermiques homogènes réalisés à haute température. Peu de } \\
\text { pertes de métal. }\end{array}$ \\
\hline & 2180.3 & & $71 \times 50 \times 31$ & 108 & $\begin{array}{l}\text { Cycles thermiques homogènes réalisés à haute température. Peu de } \\
\text { pertes de métal. Petit culot. }\end{array}$ \\
\hline & 2194.1 & Demi-produit & $40 \times 37 \times 19$ & 101 & $\begin{array}{l}\text { En cours d'utilisation. Epuration d'asséchement réalisée mais } \\
\text { conserve encore des porosités. Constitution : ferritique (50\%) et } \\
\text { aciéréc ( } 50 \% \text { eutectö̈de). }\end{array}$ \\
\hline & 2194.2 & $\begin{array}{l}\text { Objet à double } \\
\text { tranchant }\end{array}$ & $50 \times 46 \times 10$ & 80 & $\begin{array}{c}\text { En cours de confection. Très grande qualité de fabrication. } \\
\text { Constitution : âme aciérée ( } 90 \% \text { dont } 50 \% \text { eutectoïde) et contour } \\
\text { ferritique ( }(10 \%) \text {. }\end{array}$ \\
\hline & 2194.3 & $\begin{array}{l}\text { Outil tranchant } \\
\text { pour travailier la } \\
\text { pierre (chasse) }\end{array}$ & $48 \times 32 \times 16$ & 82 & $\begin{array}{l}\text { En cours de confection. Très grande qualité de fabrication avec } \\
\text { notamment une soudure en gueule de loup et une trempe } \\
\text { exceptionnelles. Constitution : acier eutectö̈de trempé }(90 \%) \text {. }\end{array}$ \\
\hline & 2208.1 & Couteau & $91 \times 14 \times 5$ & 19 & $\begin{array}{l}\text { Quclques lignes d'inclusions ou de porosités dans le sens du } \\
\text { corroyage. Qualité de fabrication proportionnnelle à la fonction de } \\
\text { l'objet. Constitution : entièrement aciérée (eutectö̈de) avec un } \\
\text { tranchant décarburé et écroui. }\end{array}$ \\
\hline 3D1 & 3342.1 & Culot de forge & $120 \times 100 \times 55$ & 707 & $\begin{array}{l}\text { Cycles thermiques courts et hétérogènes réalisés à basses } \\
\text { températures. Beaucoup de pertes de métal sous forme de fragments } \\
\text { et de fillaments. }\end{array}$ \\
\hline
\end{tabular}

Tab. 2 : Récapitulatif des analyses métallographiques pratiquées sur les ćchantillons du Roc de Pampelune (Argelliers, Hérault). Table 2: Summary of the metallographic analyses practised on the samples of the Roc de Pampelune site (Argelliers, Hérault).

\footnotetext{
${ }_{5}^{4} \mathrm{La}$ fayalite est le silicate de fer (de formule $\mathrm{Fe}_{2} \mathrm{SiO}_{4}$ ) lc plus fréquent dans les scories (d'après Mangin, 2005).

5 La wustitc est la forme cristallisée de $\mathrm{FeO}$, métastable à basse température (en dessous de $570^{\circ} \mathrm{C}$ ). Elle est très rare dans la nature, mais c'cst un constituant fréquent dans les scorics (d'après Mangin, 2005).

${ }^{6}$ Les battitures sont des particules détachécs de la surface du métal lors du martelage. Il s'agit en général d'oxydes de fer qui se sont formés à la surface du fer chaud en contact avec l'air (d'après Mangin, 2005).
} 


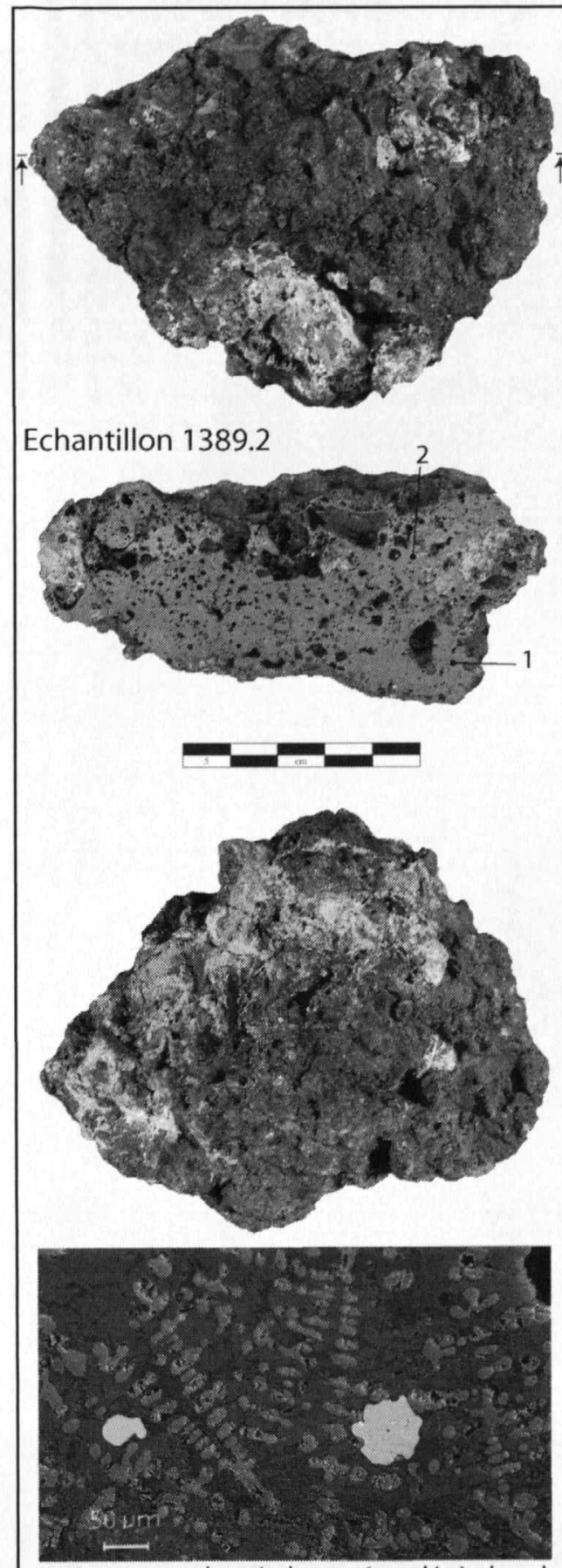

1 : Fragments de métal non réoxydés à chaud dans une matrice fayalitique à pavés avec des dendrites de wustite (Cliché UMR 5060).

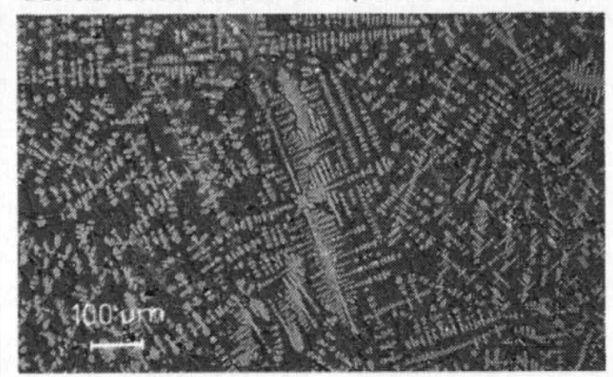

2:Dendrites de wustite dans une matrice fayalitique à lattes (Cliché UMR 5060). (bonne propreté inclusionnaire, compactage élevé), parfois encore écrouis et principalement ferritiques. Cette même configuration macro et microscopique se retrouve dans le culot prélevé dans les horizons de la forge implantée au dessus, dans un bâtiment (3D1) proche de l'église (échantillon 3342.1) (tab. 2).

\subsection{Analyse métallographique des scories du dépotoir métallurgique $2 A 3$}

La cour $2 \mathrm{~A} 3$ est un espace métallurgique plus cohérent rassemblant principalement les déchets et les vestiges de cette activité artisanale (2A3 : $69 \%$ de scorie, $15 \%$ de céramique, $11 \%$ d'os et coquillage, $3 \%$ d'objet en fer et $1 \%$ de verre), il est donc permis d'analyser simultanément les scories et les objets en fer. Tout d'abord, les 3 culots étudiés montrent des structures et des textures équivalentes avec une matrice fayalitique très homogène où se trouvent de la wustite sous forme dendritique et très peu de pertes en métal principalement non réoxydées à chaud (échantillons 2180.1,2,3). Ensuite, les 3 fragments de métal analysés présentent une constitution homogène se traduisant par un bon compactage et une épuration correcte. Leur structure, identique, est toujours aciérée (entre 0,4 et $0,8 \%$ de carbone) (échantillons 2194.2, 3 ; 2208.1). Les soudures sont très bien réalisées à l'aide de grains de sable encore visibles, dont l'utilisation permet de limiter la formation d'oxydes sur les surfaces assemblées. Elles prennent localement des formes complexes avec des assemblages en gueule de loup (fig. 6 et 7). Le travail en cours de ces 3 pièces témoigne également de la fabrication de tranchants soit écrouis soit trempés, suivant qu'il s'agisse d'un couteau ou d'un outil de type chasse $^{7}$ destiné à tailler la pierre (tab. 2).

Dix petits fragments de bronze ont été découverts dans ce même espace métallurgique. Souvent découpés, ils se présentent principalement sous la forme de lamelles. Si leur existence soulève le problème de la polymétallurgie, elle pourrait aussi indiquer le travail d'alliage cuivreux en relation avec celui du fer, car cette activité se retrouve dans un autre atelier plus spécialisé établi dans un appentis (1D4) associé à une maison dominante du centre de l'agglomération (tab. 2 et fig. 2). Ici, les vestiges sont plus diversifiés (foyer, scories cuivreuses, lamelles, plaques et fils) et plus nombreux.

\subsection{Analyse métallographique des demi-produits métal- liques}

Un volet important de notre recherche concerne la matière première utilisée par les forgerons, également appelée demi-produit lorsqu'elle est mise en forme. $\mathrm{Si}$

6 Fig. 5 : Exemple d'une étude métallographique d'un culot de forge. Fig. 5: Example of a metallographic examination of an ingot solidified at the bottom of the smelting furnace.

\footnotetext{
${ }^{7}$ La chasse du tailleur de pierre est une tige de fer terminée à l'une de ses extrémités par un large méplat destiné à chasser les aspérités sous le choc d'un maillet. Elle est généralement utilisée pour approcher les arêtes. Elle est donc souvent employée dans la confection de blocs de pierre grossièrement équarris.
} 


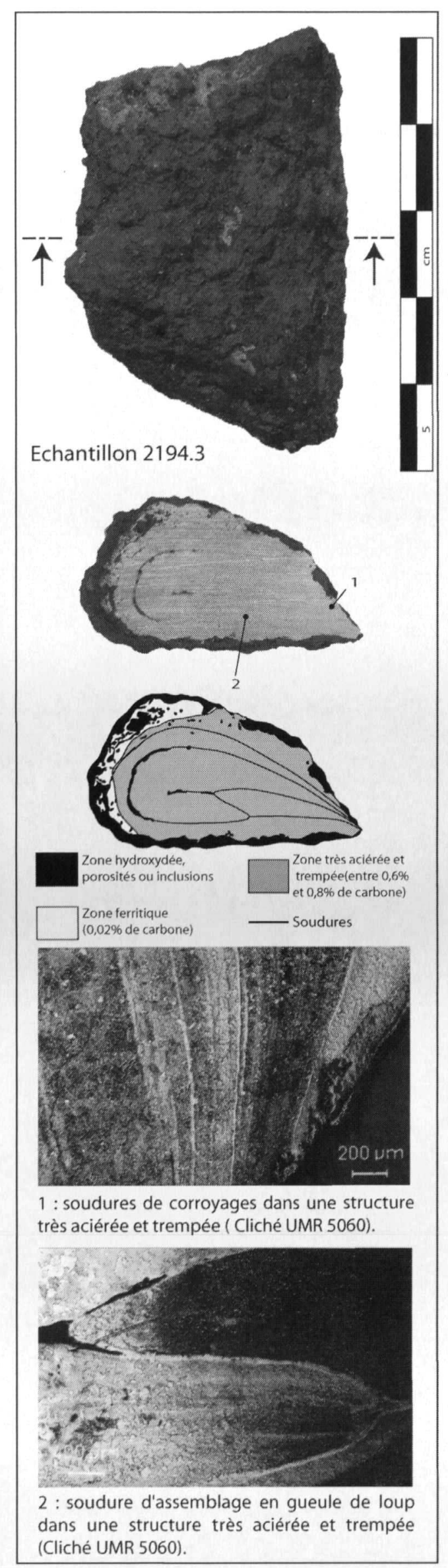

Fig. 6 : Exemple d'une étude métallographique d'un outil tranchant en cours de confection.

Fig. 6: Example of a metallographic examination of a cutting tool in the process of being made.

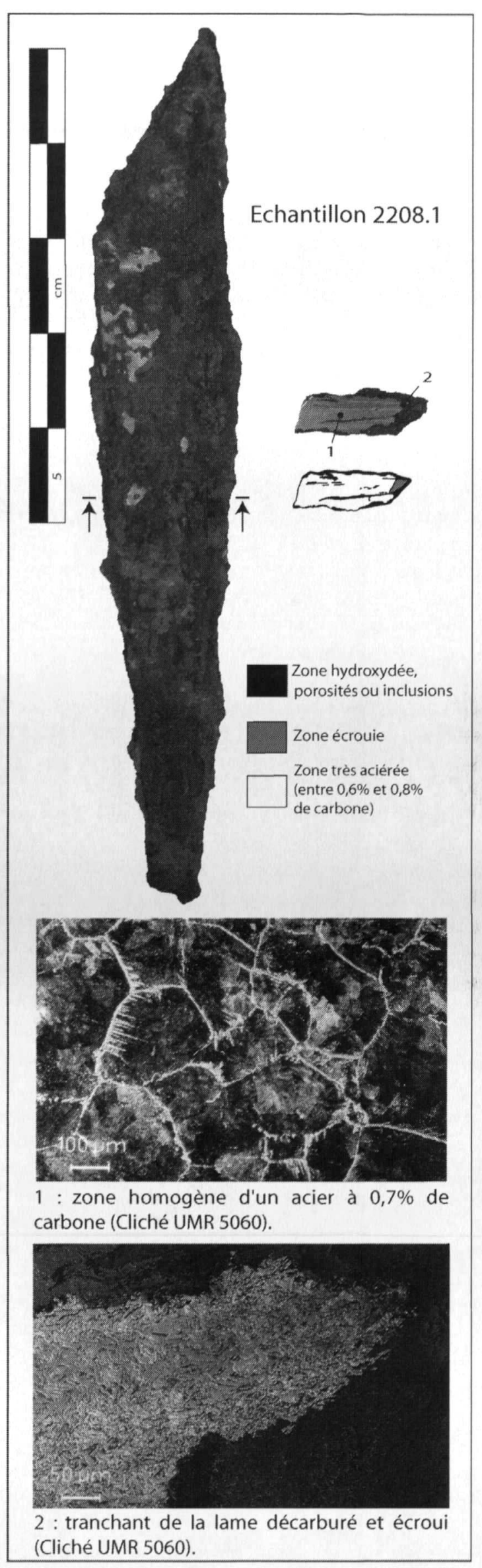

Fig. 7 : Exemple d'une étude métallographique d'un couteau. Fig. 7: Example of a metallographic examination of a knife. 
7 pièces de ce type ont été identifiées sur le site, 4 ont été étudiées à l'aide des procédés métallographiques et montrent deux cas de figure distincts (fig. 2).

L'un prélevé dans les couches de fonctionnement de 1'atelier de forge de la pièce $1 \mathrm{~L} 1$ forme un spécimen unique très proche d'un fragment de massiau ${ }^{8}$ (échantillon 1176.1 : $75 \times 52 \times 27 \mathrm{~mm}$ pour $190 \mathrm{~g}$ ). Sa section dévoile en effet une texture typique de fragment de loupe en cours de compactage : nombreux replis plus ou moins gros, zones de chapelets métalliques en cours d'agglomérations, inclusions fortement siliceuses et charbon de bois (Fluzin, 2002 ; Fluzin et al., 2000). Il comporte une structure d'acier eutectoïde décarburée en périphérie par un long séjour dans le foyer visible dans son enrobage de scorie (fig. 9). Sa forme primitive peut donc être bouleversée, mais elle ne conserve aucun reliquat de plan de frappe.

Les 6 autres demi-produits ont une morphologie plus ou moins standard en forme de barre de section rectangulaire (environ $160 \times 25 \times 10 \mathrm{~mm}$ pour $330 \mathrm{~g}$ ) ou de bloc

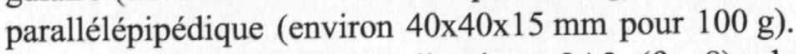
Provenant de la cour métallurgique 2A3 (fig. 8), de l'épandage domestique où le poinçon en fer a été découvert (2L) et d'une maison périphérique aux activités métallurgiques, 3 d'entres eux ont été étudiés (fig. 2). Ils dévoilent une constitution équivalente, homogène, principalement aciérée (entre 0,4 et $0,8 \%$ de carbone), fortement compactée avec peu d'inclusions.

\section{Le travail du fer : un artisanat sectorisé et spécialisé}

\subsection{Un atelier fondé sur le travail de petits objets en fer}

L'atelier de forge implanté dans un bâtiment (3D1) du quartier sommital proche de l'église recèle peu de scories, mais les culots de forge qu'il livre présentent des similitudes morphologiques et métallographiques importantes avec ceux découverts en dessous dans l'espace $1 \mathrm{H}$. La majorité des déchets produits par cette forge semble donc avoir été rejetée dans un dépotoir polyvalent, localisé en contrebas contre un rempart abandonné qui sert alors de mur terrasse $(1 \mathrm{H})$. Cette gestion des déchets est dictée par les contraintes topographiques des lieux, qui n'autorisent, au regard de l'exiguîté de l'espace sommital organisé de plus autour de l'église baptismale, qu'un rejet dans les espaces inférieurs.

La caractérisation de la production est délicate, car les rebus métallurgiques sont mélangés aux rebus domestiques de l'ensemble du quartier sommital. L'analyse métallographique des culots de forge (supra) permet cependant de discerner les grandes tendances de cette activité qui apparaît, à travers les nombreuses pertes métalliques parfois encore écrouies, principalement fondée sur des éléments plats de petites dimensions en fer (ferrite : $0,02 \%$ de carbone). Pour les façonner et les souder, ceux-ci étaient travaillés sous des cycles thermiques moyennement élevés, fréquents et de faibles amplitudes.

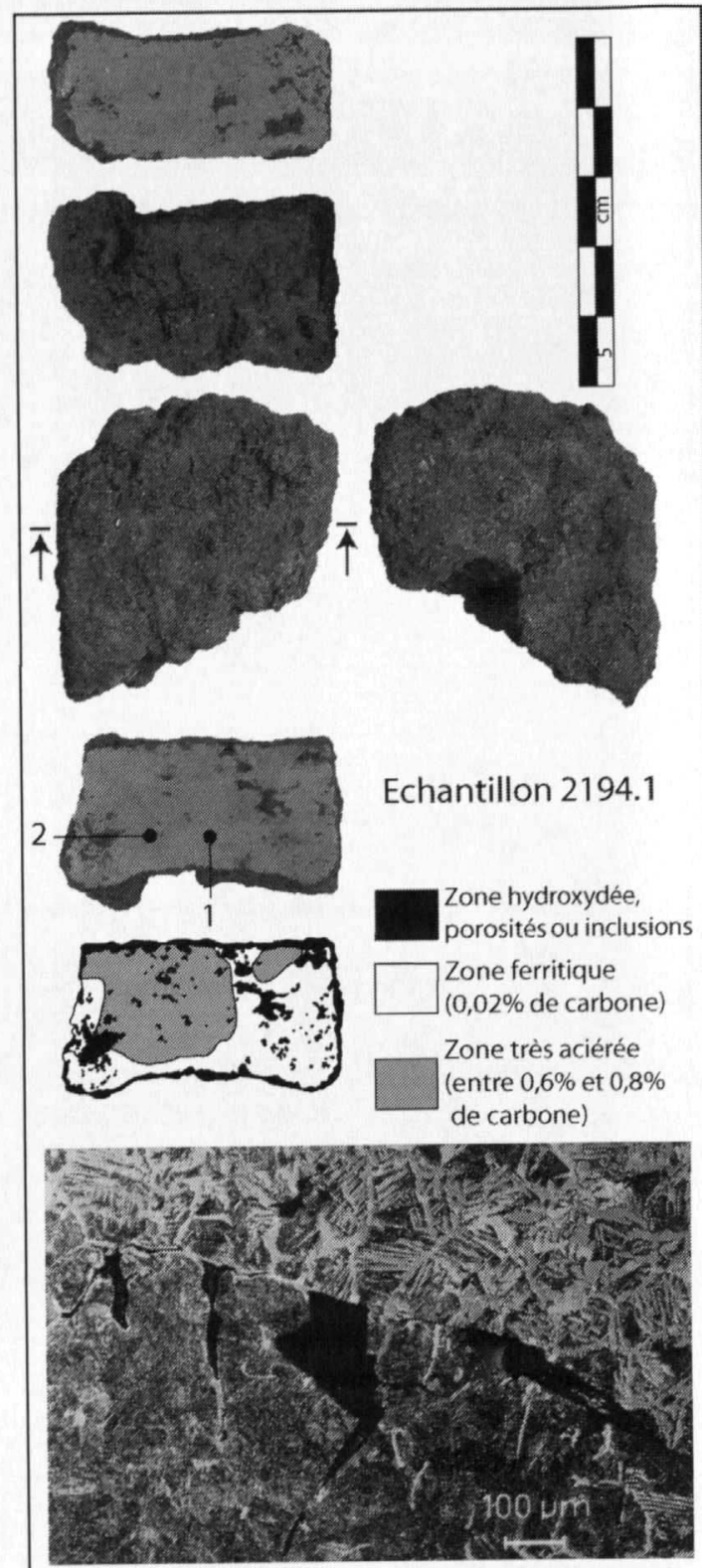

1 : zone de limite franche entre une partie très aciérée et une autre moins carburée avec des porosités aux angles vifs (Cliché UMR 5060).

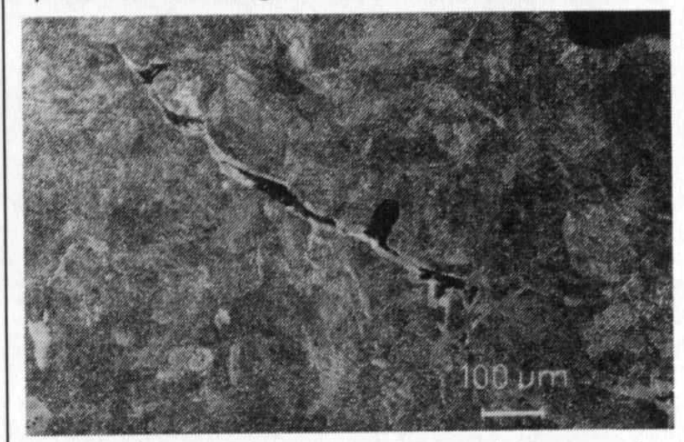

2 : acier très carburé (eutectoïde) avec des porosités très écrasées comportant une légère décarburation (Cliché UMR 5060).

Fig. 8 : Exemple d'une étude métallographique d'un demi-produit en cours d'utilisation.

Fig. 8: Example of a metallographic examination of a half-product during use.

\footnotetext{
${ }^{8}$ Massiau ou loupe : masse brute issue de la réduction directe du minerai de fer et constituée d'un métal hétérogène contenant de très nombreuses porosités et inclusions. Elle doit nécessairement être épurée pour servir de matière première.
} 


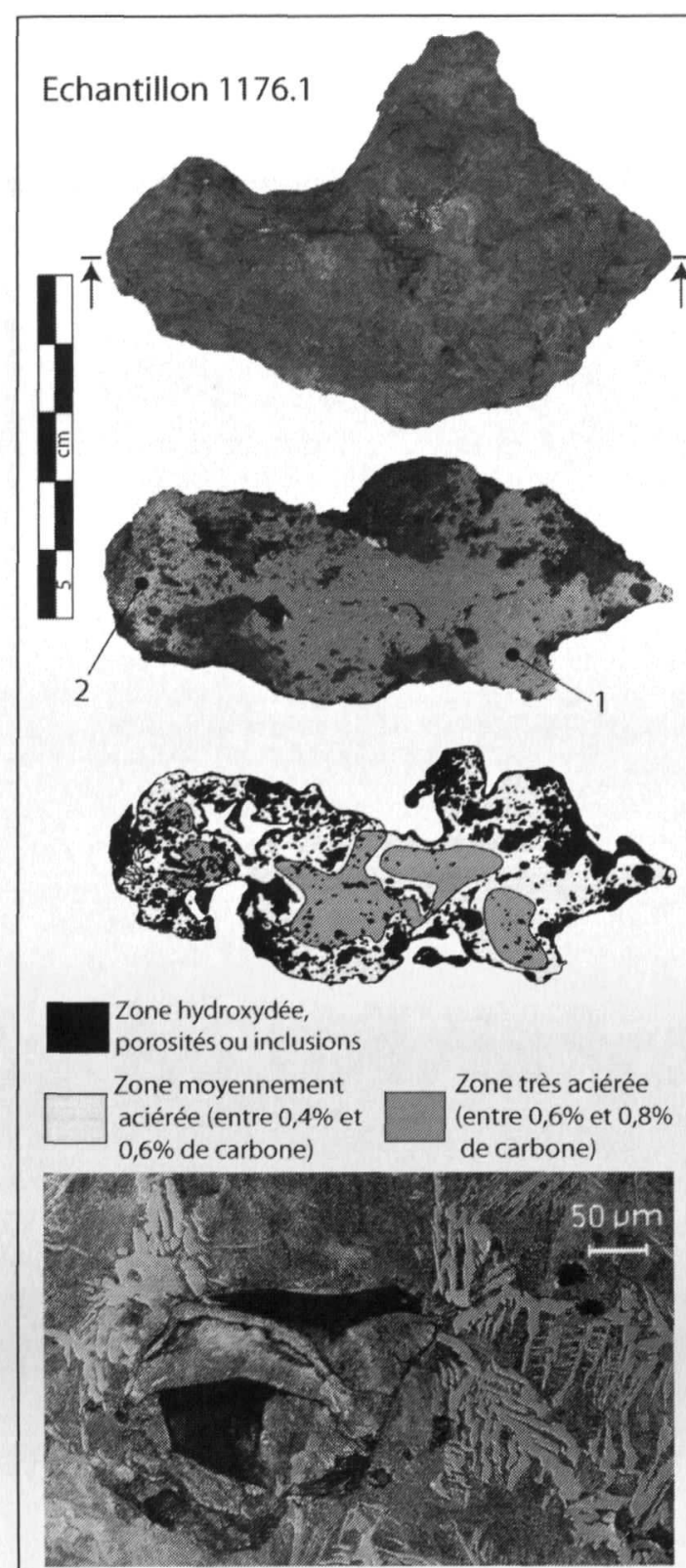

1 : gros repli dans une zone très aciérée (Cliché UMR 5060).

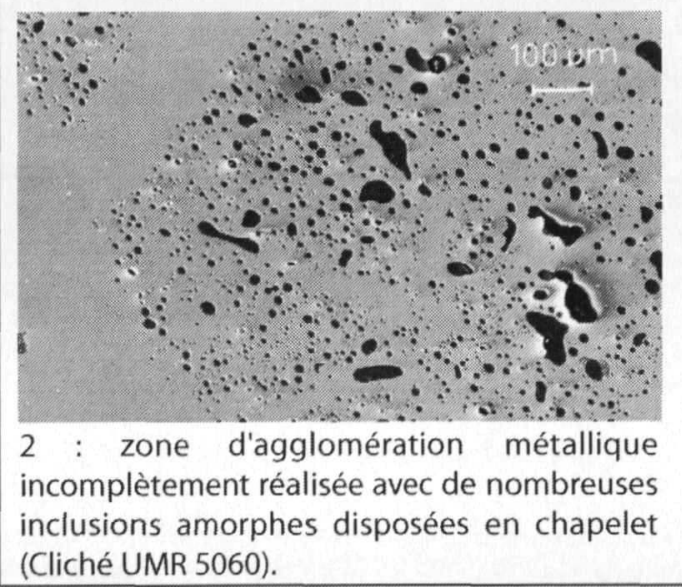

Fig. 9 : Exemple d'une étude métallographique d'un demi-produit en cours de confection à partir d'un fragment de loupe.

Fig. 9: Example of a metallographic examination of a half-product in the process of smelting from a fragment of impure ore.

\subsection{La production d'outils en acier}

L'atelier de forge localisé à l'autre extrémité de l'agglomération, dans la cour 2A3, livre un lot de scories et de rebus métalliques cohérents. Toutefois, sa production ne semble pas s'accorder avec les reliquats du foyer de forge découverts sous ce comblement quasi-exclusivement métallurgique. Il se pourrait donc que les vestiges de la structure de chauffe correspondent à un état antérieur de l'atelier de forge, dans un second temps déplacé à proximité. Tous les autres ateliers de forge sont d'ailleurs localisés dans un bâtiment comme le préconise la tradition métallurgique (Mangin, 2004) ; celui-ci devait donc être également préférentiellement abrité. La construction clôturant la cour immédiatement au sud paraît la plus adéquate pour accueillir cette forge, mais arasée par l'érosion et bouleversée par une réoccupation aux XII-XIII ${ }^{\mathrm{e}}$ siècles, elle ne livre aucun vestige susceptible de confirmer cette hypothèse, mises à part quelques scories. En revanche, la partie occidentale du bâtiment voisin, construit dans l'angle du rempart (2B1), accueille un foyer circulaire de $30 \mathrm{~cm}$ de diamètre, dont la moitié du pourtour est protégé par un muret de blocs rubéfiés en grès. Sa fouille ancienne, conduite alors que le dépotoir métallurgique (2A3) n'était pas encore connu, ne permet pas de le caractériser clairement comme un foyer de forge mais révèle une proximité topographique susceptible de mettre en évidence a posteriori sa fonction métallurgique (fig. 2).

Le dépotoir métallurgique de la cour $2 \mathrm{~A} 3$ renferme à côté des scories, 98 clous, 53 chutes de forgeage ${ }^{9}, 3$ couteaux et 3 outils tranchants en cours de confection. Leur présence indique une production polyvalente qui concerne des objets en métal de la vie courante en premier lieu représentés par les clous. Elle se distingue cependant par la confection d'objets en acier tranchants (couteaux, ciseaux à bois et à pierre...) traités, selon les analyses métallographiques, soit par écrouissage soit par traitement thermique (trempe) (supra, fig. 6 et 7). Selon le classement typologique des scories et leur analyse métallographique, les cycles thermiques associés sont élevés, longs et de faibles amplitudes. Ils sont également caractérisés par des pertes en oxyde et en métal peu importantes, significatives de cycles de chauffe maîtrisés et adaptés à la matière travaillée.

\subsection{Le travail d'une matière première standardisée}

L'analyse métallographique d'un demi-produit provenant de l'atelier précédemment traité (2A3) révèle une confection des objets à partir d'une matière première déjà compactée et épurée dont 6 exemplaires ont été découverts à divers endroits sur le site (supra, fig. 8). Parallèlement, l'analyse métallographique d'un autre élément mis au jour dans l'atelier de la pièce $1 \mathrm{~L} 1$ apporte des éléments de réflexion sur la formation de ces demi-produits aux formes plus ou moins standardisées en petit bloc (environ $40 \times 40 \times 15 \mathrm{~mm}$ ) ou en petite barre (environ $160 \times 25 \times 10 \mathrm{~mm}$ ) principalement aciérés (entre 0,4 et

\footnotetext{
${ }^{9}$ Les 53 chutes de forgeage sont principalement représentées par des petites plaques parallélépipédiques en fer d'environ un millimètre d'épaisseur n'excédant que rarement les $5 \mathrm{~cm}^{2}$. Le reste du lot comporte majoritairement des petites tiges.
} 
$0,8 \%$ de carbone). L'existence d'un fragment de loupe en cours de mise en forme pourrait en effet signifier que la matière première utilisée sur le site ne provient pas de demi-produits prêts à l'emploi, mais de fragments de massiau légèrement manufacturés dont il faut encore réaliser l'épuration, le compactage et la mise en forme (supra, fig. 9).

Cet approvisionnement en matière première dans une phase haute de la chaîne opératoire pourrait indiquer la proximité d'un centre de réduction, mais également suggérer qu'une partie de la production des demi-produits est destinée à l'exportation. La détermination de l'origine de la matière première constitue donc une piste à suivre, d'autant qu'au pied du site s'étend un gîte affleurant de minerai pisolithique permettant d'envisager l'hypothèse de réductions liées à l'occupation du Roc de Pampelune (s.n. 1971).

\section{En guise de conclusion : un village de métallurgiste ?}

La chronologie resserrée de l'occupation du Roc de Pampelune - de la fin du $\mathrm{V}^{\mathrm{c}}$ siècle au milieu du VI siècle - et la diversité des travaux mis en évidence dans les 3 ateliers de forge identifiés incitent à considérer leur fonctionnement synchrone et durable. Parallèlement, la mise en forme de fragment de loupe, le peu de pertes au feu observées et l'élaboration d'outils parfois complexes montrent l'existence de forgerons qualifiés aux savoirfaire élevés exerçant leur compétence dans le cadre d'une production spécialisée et sectorisée. Ces témoignages mettent en évidence un déséquilibre entre la qualité et la masse du travail produit et la nature et la taille de l'établissement qui livre peu d'aménagements agricoles. Pour l'heure, la population est donc davantage perçue comme un regroupement d'artisans spécialisés dans les arts du feu que comme une petite communauté agricole. La présence d'autres métallurgistes travaillant les alliages cuivreux dans un appentis (1D4) associé à une maison dominante du centre de l'agglomération (fig. 2), mais aussi dans des secteurs du rempart septentrional (2A3 et $1 \mathrm{M} / 1 \mathrm{~N})$ vient corroborer cette vision et soulève non seulement le problème de la polymétallurgie, mais encore d'une pratique plus large des arts du feu rattachée également à la présence possible de verriers.

Ces dichotomies posent la question de l'envergure de la diffusion de ces ateliers spécialisés qui seraient, à côté des besoins propres de la bourgade, amenés à exporter au moins à moyenne distance. Cette hypothèse déjà suggérée par la possible production de demi-produits est soutenue par l'insertion rapide de cette petite agglomération de fondation récente dans les grands circuits du commerce méditerranéen (Schneider, 2005).

Le rôle des ressources minières et surtout forestières locales, en cours d'évaluation, doit alors être pris en compte, car ces dernières constituent l'une des principales pistes susceptibles d'expliquer l'investissement qui a été consenti lors de la création de cette bourgade fortifiée située en marge de l'ancien système du peuplement local.

En Gaule du Sud, l'exemple du Roc de Pampelune témoigne de la persistance d'un artisanat spécialisé durant
l'Antiquité tardive, notamment établi dans ces nouveaux villages perchés. Ils peuvent témoigner, en parallèle d'une fonction administrative et religieuse, d'une concentration nouvelle d'une main-d'œuvre spécialisée et illustrent en cela les capacités d'investissements économiques, techniques et intellectuels d'une société en mouvement (Schneider, 2006 sous presse).

\section{Bibliographie}

BREICHNER, H., CHABAL, L., LECUYER, N. et SCHNEIDER, L., 2002 - Artisanat potier et exploitation du bois dans les chĉnaies du nord de Montpellier au XIIle s. (Hérault, Argelliers, Mas-Vicl). Archéologie du Midi Médiéval, 20 : 57-106.

Cammas, C., Chanpagne, F., DaVId, C., DeSaCHY, B. et GUYARD, L., 1995 - Le problème des «terres noircs» sur les sites urbains tardo-antiques et médićvaux : réflexions et propositions méthodologiques à partir de l'exemple des fouilles du Collège de Francc à Paris. Les nouvelles de l'archéologie, 61 : 22-29.

DUVAUChELLE, A., 2005 - Les outils en fer du Musée romain d'Avenches, Association Pro Aventico, Avenches, Documents du Muséc Romain d'Avenches, $\mathrm{n}^{\circ} 11$.

FLUZIN, P., 1983 - Notions élémentaires de sidérurgic. Métallurgies Africuines. Nouvelles contributions. Mémoire de la Société des Africanistes, $9: 13-44$.

FLUZIN, P., 2002 - La chaîne opératoire en sidérurgie : matériaux archéologiques et procédés. Apport des ćtudes métallographiques. In H. Bocoum (Dir.) Aux origines de la métallungie du fer en Afrique. Une Ancienneté méconnue. Afrique de l'Ouest et Afrique centrale, U.N.E.S.C.O., Paris, 59-91.

FLUZIN, P., PLOQUIN, A. et SERNEELS, V., 2000 - Archéométric des déchets de production sidćrurgique. Moyens et méthodes d'identification des différents éléments de la chaîne opératoire dirccte. Gallia, $57: 101-121$.

GUYARD, L., 2003 - Le Collège de France (Paris). Du quartier galloromain au Quartier latin, Maison des Sciences de l'Homme, Paris, Documcnts d'Archćologic Françaisc, $n^{\circ} 95$.

MANGIN, M. (Dir.), 2004 - Le fer. Errance, Paris. Collection «Archéologiques".

PELLECUER, C., 2000 - La villa des Prés-Bas (Loupian, Hérault) dans son environnement. Contribution à l'étude de la villa et de l'économie domaniale en Narbonnaise, Université de Provence AixMarseille I, France.

s.n., 1971 - Montpellier, Saint-Martin de Londres. Carte géologique détailléc de la France (XXVII-42). Bureau des Recherches Géologiques et Minières.

SCHNEIDER, L., 2003 - Nouvelles recherches sur les habitats de hauteur de l'Antiquité tardive et du haut Moyen Age dans le sud-est de la France : Le Roc de Pampclune à Argellicrs (Hérault). Les nouvelles de l'archéologie, $92: 9$-16.

SCHNEIDER, L., 2005 - Cité, castrum et «pays» : espace et territoires en Gaule méditerranéenne durant le haut Moyen Agc. L'exemple de la cité de Nîmes et du pagus de Maguclone (V-XIe s.). In P. Cressier. Bacza (Dir.) El Castillo y La Ciudad. Espacios y Redes (ss. VI-XIII), Espagne, septembre 2002, Actes du colloque Castrum 8. Casa de Velazquez, Madrid.

SCHNEIDER, L., 2006 (sous presse) - Rythmes de l'occupation rurale et formes de l'habitat dans le sud-est de la France entre Antiquité et Moyen Age (IVe-VIIIe s.). Etat possible de la recherche et nouvelles interrogations. Gallia.

SERNEELS, V., 1998 - La chaîne opératoire de la sidérurgie ancienne. In M. Feugc̀re, V. Scmccls (Dir.) Rechenches sur l'économie du fer en Méditerranée nord-occidentale, Monique Mergoil, Montagnac, 7 44. Collection «Monographies instrumentum $n^{\circ} 4$ ». 